\title{
A MVSA Approach to Mine Information from APT Data
}

C. M. Parish ${ }^{1 *}$, C. Capdevila ${ }^{2}$, and M. K. Miller ${ }^{1}$

${ }^{1}$ - Materials Science and Technology Division, Oak Ridge National Laboratory, Oak Ridge, TN 37831, USA

${ }^{2}$-Centro Nacional de Investigaciones Metalúrgicas (CENIM-CSIC), Madrid, E-28040, Spain

*parishcm@ornl.gov

Multivariate statistical analysis (MVSA) methods, such as used in analytical electron microscopy and surface science, were applied to atom probe tomography (APT) data. It was found that MVSA methods allow fast and intuitive interpretation of APT data, where both spatial and spectral variance are described simultaneously in an unbiased manner. In this work, a FeCr-Al-Ti alloy $\left(\mathrm{PM} 2000^{\mathrm{TM}}\right)$ subjected to different heat treatments was examined via APT. The different scale three-phase microstructures resulting from these heat treatments provided an interesting test of the capabilities and limits of the present MVSA methodology. Principal component analysis (PCA)-based MVSA identified the precipitate populations in all three samples, including ultrafine precipitates with the use of small $(0.5 \mathrm{~nm})$ voxel size, and high resolution $(0.02 \mathrm{u})$ mass binning.

\section{Introduction}

Atom probe tomography (APT) produces large datasets that are resolved in both the spatial and isotopic (i.e., mass-to-charge state) domains [1-3]. A constant challenge when analyzing APT data is to interpret and quantify the parameters of the microstructural features in 
the large datasets in a way that is straightforward, intuitive, unbiased, and provides microstructural insight to the problem being investigated.

This same problem has also been encountered in the analytical electron microscopy (AEM) and surface science communities, where large spectrum image (SI) datasets are acquired by analytical methods, such as energy dispersive X-ray spectroscopy, electron energy loss spectroscopy, cathodoluminescence, X-ray photoelectron spectroscopy, secondary ion mass spectroscopy, and other techniques. In a SI, an entire detector response spectrum is recorded at each spatially resolved pixel or voxel in the region of interest. Although generally smaller in size relative to APT datasets, these SIs are still large on the absolute scale. The solution invoked to mine chemical, elemental, and microstructural information from the data acquired using these applications is multivariate statistical analysis (MVSA) [4-9], generally bilinear factor methods based upon principal component analysis (PCA) [10-11].

SI datasets are "unfolded" from two or three spatial dimensions, plus a spectral dimension, into a matrix $\mathbf{D}$, and then approximated by a low-rank bilinear factor model consisting of score images (eigenimages) A and loading spectra (eigenspectra) S:

$$
\mathbf{D} \approx \widehat{\mathbf{D}}=\mathbf{A} \mathbf{S}^{\mathrm{T}}
$$

Here, $\mathbf{D}$ is the original data, $\widehat{\mathbf{D}}$ the low-rank approximation, and $\mathbf{A} \mathbf{S}^{\mathrm{T}}$ the bilinear model. $\mathbf{D}$ and $\widehat{\mathbf{D}}$ have dimensions $\mathrm{n}_{\text {voxels }} \times \mathrm{n}_{\text {channels }}$, $\mathbf{A}$ has dimensions $\mathrm{n}_{\text {voxels }} \times \mathrm{p}$, and $\mathbf{S}$ has dimensions $\mathrm{n}_{\text {channels }} \times$ $\mathrm{p}$, where $\mathrm{p}$ is the number of factors or components retained. $\mathbf{S}^{\mathrm{T}}$ denotes the matrix-transpose of S. As written, Equation (1) is a generic bilinear factor model. If each column of A is mutually orthogonal, and each column of $\mathbf{S}$ is mutually orthogonal and the components are sorted in order of decreasing variance, then Equation (1) is the PCA solution to $\mathbf{D}$, and the value p is referred to 
as the number of principal components (PCs). When dealing with discrete data following Poisson statistics, such as ion hits, it is important to perform Poisson-scaled PCA [5, 12-15].

Previous work [16-17] has shown with mathematical and computational detail how Poisson-scaled PCA and related MVSA methods can be successfully applied to APT datasets by treating the APT datasets simply as spectrum image data. In this paper, these new techniques will be applied to a practical materials problem by analyzing APT data from a Fe-Cr-Al-Ti alloy heat-treated to produce progressively smaller precipitate sizes, are examined to test the viability of using MVSA to find the variance associated with the different precipitate size regimes.

\section{Materials and Methods}

\subsection{Atom probe tomography}

Samples of a PM2000 ${ }^{\mathrm{TM}}$ Fe-Cr-Al-Ti alloy were aged for $3600 \mathrm{~h}$ at three temperatures, 400, 435, and $475^{\circ} \mathrm{C}$, heat-treatments known [18] to provide different size populations and distributions of Cr-rich $\alpha^{\prime}$ and $\mathrm{Fe}(\mathrm{Ti}, \mathrm{Al})$-rich $\beta^{\prime}$ precipitates. Atom probe tomography specimens were prepared by electropolishing followed by annular FIB-milling. APT analyses were performed in the voltage-pulsed mode with a Cameca Instruments Laser-LEAP 4000X HR. The APT data was converted to position files using standard reconstruction methods. The Fe-Cr-AlTi specimens analyzed and dataset reconstruction parameters are summarized in Table I.

\section{$\underline{2.2 \text { Multivariate statistical analysis }}$}

The details of the MVSA method are discussed elsewhere [16-17]. In brief, a 4dimensional spectrum image datacube is generated, consisting of voxels in $(\mathrm{x}, \mathrm{y}, \mathrm{z})$ and an $\mathrm{m} / \mathrm{n}$ mass spectrum associated with each voxel. The position file of the APT reconstruction is 
interrogated one ion at a time, and each ion is assigned to the ([x,y,z],m/n) data element in the SI that matches its location and $\mathrm{m} / \mathrm{n}$ state. This is conceptually no different from binning $\mathrm{X}$-rays, secondary ions, etc., into an SI datacube acquired via AEM or surface analysis [19-20].

The APT datacube is pre-scaled for Poisson noise using the standard method from the AEM literature [5, 12-15] and then subjected to singular value decomposition (SVD) [21-24]. By definition the squares of the singular values are the eigenvalues, which are sorted into descending order and plotted semi-logarithmically vs. the eigenvector index. The resulting plot will have a knee between the semi-logarithmic background and the first few components. The location of this knee denotes the number of significant principal components. As a result of the small number of ions identified per voxel (mean $\sim 4-5$ ions/voxel), the signal/noise levels were not always sufficient to resolve the precipitate populations. A previously published technique [25] from the X-ray spectrum imaging literature is invoked to increase the signal/noise, where voxels are binned by a factor of $2 \times 2 \times 2$ or $4 \times 4 \times 4$, prior to singular value decomposition (SVD), followed by up-sampling of the SVD-determined loading spectra to the original data resolution. After SVD, the data was subjected to spatial-simplicity matrix rotations [24]. Spatially-simple score images were smoothed by a $3 \times 3 \times 3$ voxel cubic moving average (MATLAB command 'smooth3') and isoscore surfaces were rendered. All APT data analysis, MVSA, and visualization were performed using MATLAB (The Mathworks, Inc., Natick, MA, USA; R2010a V7.10.0.499) routines written by the authors.

Previously [16-17], coarse mass-spectral resolutions, 0.10 - $0.16 \mathrm{u} / \mathrm{bin}$, were used to minimize the amount of computer memory needed at reasonable voxels sizes, such as $(0.5 \mathrm{~nm})^{3}$ to $(2 \mathrm{~nm})^{3}$. In the present study, the previously reported methodologies have been refined to utilize a sparse data handling technique, where only non-zero elements (i.e., ion locations) are 
stored in computer memory. The APT dataset for the alloy heat-treated at $435^{\circ} \mathrm{C}$ for $3600 \mathrm{~h}$, for example, would require 20 Gb of computer memory at a $(0.5 \mathrm{~nm})^{3}$ voxel size and $1851 \mathrm{~m} / \mathrm{n}$ channels per voxel. By utilizing sparse data handling, this same data is stored in $<1 \mathrm{~Gb}$ of computer memory, resulting in much finer spectral resolutions (0.02 u in this work) at small voxel sizes, $(0.5 \mathrm{~nm})^{3}$. At this voxel size, the typical number of ions per voxel was $\approx 5$. With $1851 \mathrm{~m} / \mathrm{n}$ channels per voxel, the SI datacube is $\approx 99.7-99.9 \%$ sparse. For the study presented here, the APT reconstructions were converted to sparse SIs with (0.5 nm)-cubed voxels and 0.02 u spectral spacing over the range 3-40 u, totaling 1851 channels.

\section{Results}

The APT dataset for the Fe-Cr-Al-Ti alloy heat treated at $475^{\circ} \mathrm{C}$ for $3600 \mathrm{~h}$ will be used to illustrate the detailed steps of the MVSA method used to characterize the precipitates, whereas for the two alloys heat-treated for $3600 \mathrm{~h}$ at $400^{\circ} \mathrm{C}$ and $435^{\circ} \mathrm{C}$ only the final rendered isoscore surfaces are presented. A standard isoconcentration-surface rendering of the $475^{\circ} \mathrm{C}$ for $3600 \mathrm{~h}$ APT dataset is shown in Fig. 1a, and did not make use of MVSA methodology. In Fig. 1b, the sum spectrum of the $475^{\circ} \mathrm{C}$ for $3600 \mathrm{~h}$ condition dataset is shown. Fe, $\mathrm{Cr}, \mathrm{Al}$, and Ti peaks are observed in the sum spectrum. The resolution used is $0.02 \mathrm{u} / \mathrm{bin}$, which is the same resolution used in the subsequent MVSA analysis. The eigenvalue-vs-eigenvector index plots for the $(0.5$ $\mathrm{nm})^{3}$ voxel and $0.02 \mathrm{u}$ data is shown in Fig. 1c. Eigenvalues for 3 different binning levels $\left(1^{3}, 2^{3}\right.$, $4^{3}$ ) are indicated on the graph. An arrow indicates that $2^{3}$ binning resolves three significant PCs. The isoconcentration surface (Fig. 1a) shows that the Cr-enriched $\alpha^{\prime}$ precipitates (cyan) are uniformly distributed within the volume, and two $\beta^{\prime}$ precipitates are observed, one of which intersects the $\mathrm{YZ}$ face of the volume. Binning by $4^{3}$ indicates a higher algebraic rank in the 
eigenvalue plot of Fig. 1c; however, inspection of these higher-order eigenimages and eigenspectra indicate artifacts such as peak shifts or random noise is being identified by the PCA computation; further work is needed for rank selection and binning level selection.

The PCA score images (left column) and loading spectra (right column) for the $475^{\circ} \mathrm{C}$ for $3600 \mathrm{~h}$ sample are shown in Fig. 2. The $1^{\text {st }} \mathrm{PC}$ (Fig. 2a) is the mean. The $2^{\text {nd }} \mathrm{PC}$ (Fig. 2b) shows precipitates assigned negative pixel scores, where the associated loading spectrum shows negative Cr-peaks and positive Fe- and Al-peaks, with $\mathrm{Ti} \sim$ zero. This is interpreted to mean that more $\mathrm{Cr}$ is present, with less $\mathrm{Fe}$ and $\mathrm{Al}$, at the locations of the precipitates in the score image of Fig. 2b. Similarly, in the $3^{\text {rd }}$ PC (Fig. 2c) the small precipitate (negative score region, designated by arrow) is associated with increased $\mathrm{Ti}$ and $\mathrm{Al}$ and decreased Fe and $\mathrm{Cr}$. Also, very small $\mathrm{Si}$ and $\mathrm{V}$ peaks are observed in the $3^{\text {rd }} \mathrm{PC}$, indicating that these minor contaminants are associated with the $\beta^{\prime}$. The precipitate is related to the $\beta^{\prime}$ precipitate intersecting the YZ face of the isoconcentration image in Fig. 1a.

Because of the correlation and anticorrelation between the positive and negative scores and loadings, PCA results, such as that shown in Fig. 2, are often difficult to interpret. The spatial-simplicity (SS) PCA representation of the PCA result is shown in Fig. 3. The $1^{\text {st }}$ SS component (Fig. 3a) shows the alloy matrix. The $2^{\text {nd }}$ SS component (Fig. 3b) shows precipitates enriched in Cr. The $3^{\text {rd }}$ SS component (Fig. 3c) shows the same precipitate designated by arrow in Fig. 2c, which is enriched in Ti and Al. A very small Mn peak is observed in the matrix component, and small Si and V peaks are seen in the $\beta^{\prime}$ precipitates.

The eigenvalue-eigenvector index plots for the (a) $435^{\circ} \mathrm{C}$ for $3600 \mathrm{~h}$ and (b) $400^{\circ} \mathrm{C}$ for 3600 h APT datasets are shown in Fig. 4, each of which were calculated with the same voxel resolution $(0.5 \mathrm{~nm})^{3}$, and spectral resolution, $0.02 \mathrm{u}$ over $3-40 \mathrm{u}$, as the $475^{\circ} \mathrm{C}$ for $3600 \mathrm{~h} \mathrm{APT}$ 
dataset. PCA followed by SS-PCA were performed on these datasets using the same procedure as for the first APT dataset.

Because the spectral differences between voxels are reduced in the finer-precipitate-size datasets the first three PCs were not the matrix and two precipitate components. A small number of artifact or noise components also appeared. These were maintained through PCA and SSPCA analysis, but were not rendered. The artifacts were essentially the same as observed in previous reports [16-17].

The rendered isoscore surfaces for the $\alpha^{\prime}$ and $\beta^{\prime}$ precipitate phases for the three APT datasets collected from the heat-treated alloy, all created with $(0.5 \mathrm{~nm})^{3}, 0.02$ u over 3-40 u, $2 \times 2 \times 2$ binning with up-sampling to the original resolution are shown in Fig. 5 The images from left to right in Fig. 5 are consistent with decreasing annealing temperature resulting in finer-scale precipitate sizes from left to right, as identified via the MVSA methodology. The comparison of the $475^{\circ} \mathrm{C}$ for $3600 \mathrm{~h}$ isoscore surface shown in Fig. 5 (with MVSA) to the isoconcentration surface in Fig. 1a (without MVSA) shows good agreement.

\section{Discussion \& Conclusions}

For the $400^{\circ} \mathrm{C}$ for $3600 \mathrm{~h}$ APT dataset (Fig. 5c), where the $\beta^{\prime}$ precipitate size is $\sim 1-2 \mathrm{~nm}$, MVSA successfully extracts the precipitate size populations accurately, with simultaneous insight into both the spatial and spectral character of the different precipitate phases via the score images and loading spectra. Although MVSA in general, and PCA-based methods in particular, are still under development for APT applications, results from this study indicate that spatial resolutions of $0.5-1 \mathrm{~nm}$ are achievable, at least in the case of elementally distinct precipitates. 
This is encouraging because many modern materials science problems require the ability to resolve extremely small features.

Additionally, these results demonstrated that the use of sparse data-handling methods allows for excellent memory-management in the MVSA computation. Large APT datasets could easily overwhelm even a high-end laboratory workstation. However, the current calculations were conducted using both high spatial resolution $(0.5 \mathrm{~nm})$ and high spectral resolution $(0.02 \mathrm{u})$. Since the highest charge-states commonly seen are $4^{+}$and $3^{+}$, a spectral overlap of $(0.33 \mathrm{u}-0.25$ u) $\sim 0.08$ u may be possible between two different ions [2], thus working with a spectral resolution of $0.02 \mathrm{u}$ should allow many common near-overlaps to be resolved. (Common isobars, such as ${ }^{58} \mathrm{Ni}^{2+}$ and ${ }^{58} \mathrm{Fe}^{2+}$, will remain unresolved.)

In short, this paper has illustrated the ability of MVSA-based methods to resolve a wide range of precipitate sizes in heat-treated PM2000 Fe-Cr-Al-Ti alloys aged at a series of different temperatures. MVSA of APT reconstructions provides intuitive and unbiased spectral and spatial insight regarding fine-scale phases present in a material, even at very high spatial and spectral resolution.

\section{Acknowledgments}

This research was conducted as part of the Shared Research Equipment (SHaRE) User Program, which is sponsored at Oak Ridge National Laboratory (ORNL) by the Scientific User Facilities Division, Office of Basic Energy Sciences, U.S. Department of Energy. PM2000 is a trademark of Plansee.

\section{References}


[1] T. F. Kelly and M. K. Miller: Atom Probe Tomography. Review of Scientific Instruments V78(03), P.031101 (2007).

[2] M. K. Miller, Atom Probe Tomography: Analysis at the Atomc Level. (Kluwer Academic, New York, 2000).

[3] D. N. Seidman and K. Stiller: An atom probe tomography primer. MRS Bulletin V34(October), P.717-721 (2009).

[4] C. Jeanguillaume and C. Colliex: Spectrum image: the next step in EELS digital acquisition and processing. Ultramicroscopy V28(1-4), P.252-257 (1989).

[5] M. R. Keenan: Multivariate analysis of spectral images composed of count data, in Techniques and Applications of Hyperspectral Image Analysis, edited by H. F. Grahn and P. Geladi (John Wiley \& Sons, Chichester, 2007), pp. 89-126.

[6] P. G. Kotula, M. R. Keenan and J. R. Michael: Automated analysis of SEM X-ray spectral images: a powerful new microanalysis tool. Microscopy and Microanalysis V9(1), P.1-17 (2003).

[7] V. S. Smentkowski, J. A. Ohlhausen, P. G. Kotula and M. R. Keenan: Multivariate statistical analysis of time-of-flight secondary ion mass spectrometry images-looking beyond the obvious. Applied Surface Science V231-232, P.245-249 (2003).

[8] J. M. Titchmarsh and S. Dumbill: Multivariate statistical analysis of FEG-STEM EDX spectra. Journal of Microscopy-Oxford V184(3), P.195-207 (1996).

[9] P. Trebbia and N. Bonnet: EELS elemental mapping with unconventional methods I. Theoretical basis: image analysis with multivariate statistics and entropy concepts. Ultramicroscopy V34, P.165-178 (1990).

[10] I. T. Jolliffee, Principal Component Analysis, 2nd ed. (Springer, New York, 2002). 
[11] E. R. Malinowski, Factor Analysis in Chemistry, 2nd ed. (Wiley, New York, 1991).

[12] M. R. Keenan: Maximum likelyhood principal component analysis of time-of-flight secondary ion mass spectrometry spectral images. Journal of Vacuum Science \& Technology A V23(4), P.746-750 (2005).

[13] M. R. Keenan and P. G. Kotula: Accounting for Poisson noise in the multivariate analysis of ToF-SIMS spectrum images. Surface and Interface Analysis V36(3), P.203-212 (2004).

[14] M. R. Keenan and P. G. Kotula: Optimal scaling of TOF-SIMS spectrum-images prior to multivariate statistical analysis. Applied Surface Science V231-232, P.240-244 (2004).

[15] M. R. Keenan, V. S. Smentkowski, J. A. Ohlhausen and P. G. Kotula: Mitigating dead-time effects during multivariate statistical analysis of ToF-SIMS spectral images. Surface and Interface Analysis V40, P.97-106 (2008).

[16] C. M. Parish and M. K. Miller: Multivariate statistical analysis of atom probe tomography data. Ultramicroscopy V110(11), P.1362-1373 (2010).

[17] C. M. Parish, C. Capdevila and M. K. Miller: Applying Multivariate Statistical Analysis to Atom Probe Tomography. Microscopy and Microanalysis V16(Suppl. 2), P.1858-1859 (2010).

[18] C. Capdevila, M. K. Miller and K. F. Russell: Aluminum partitioning during phase separation in Fe-20\%Cr-6\%Al ODS alloy. Journal of Materials Science V43, P.3889-3893 (2008).

[19] P. G. Kotula, M. R. Keenan and J. R. Michael: Tomographic spectral imaging with multivariate statistical analysis: comprehensive 3D microanalysis. Microscopy and Microanalysis V12(1), P.36-48 (2006). 
[20] V. S. Smentkowski, S. G. Ostromiski, E. Braunstein, M. R. Keenan, J. A. T. Ohlhausen and P. G. Kotula: multivariate statistical analysis of three-spatial-dimension TOF-SIMS raw data sets. Analytical Chemistry V79(20), P.7719-7726 (2007).

[21] J. W. Demmel, Applied Numerical Linear Algrebra. (Society for Industrial and Applied Mathematics, Philadelphia, 1997).

[22] G. H. Golub and C. F. Van Loan, Matrix Computations, 3rd. ed. (Johns Hopkins University Press, Baltimore, 1996).

[23] L. N. Trefethen and D. Bau, Numerical Linear Algebra. (Soceity for Industrial and Applied Mathematics, Philadelphia, 1997).

[24] M. R. Keenan: Exploiting Spatial-Domain Simplicity in Spectral Image Analysis. Surface and Interface Analysis V41, P.79-87 (2009).

[25] P. G. Kotula and M. R. Keenan: Automated analysis of large (>4Gb) spectral images with efficient out-of-core-RAM algorithms. Microscopy and Microanalysis V9(Supp 2), P.152-153 (2003).

\section{Figures}
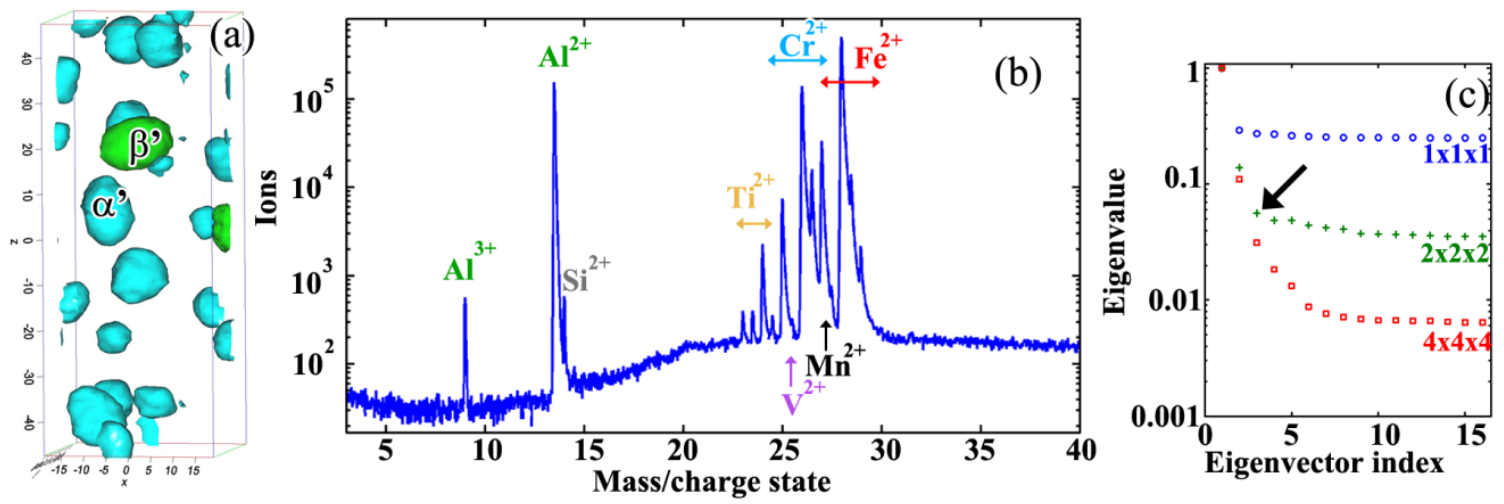
Figure 1: (a) Standard isoconcentration surfaces for $\alpha^{\prime}$ and $\beta^{\prime}$ (not MVSA results). (b) Sum spectrum, 3 to $40 \mathrm{u}, 0.02 \mathrm{u}$ bins for sample aged at $475^{\circ} \mathrm{C}$ for 3600 h. (c) Normalized eigenvalue-eigenvector index plot for the dataset, gridded into $(0.5 \mathrm{~nm})^{3}$ voxels, with the indicated $(1 \times 1 \times 1,2 \times 2 \times 2,4 \times 4 \times 4)$ level of binning. Arrow indicates 3rd significant eigenvalue (PC) with $2 \times 2 \times 2$ binning.


-2 Score 2

Figure 2: PCA of sample aged at $475^{\circ} \mathrm{C}$ for 3600 h, 3 to $40 \mathrm{u}, 0.02 \mathrm{u}$ bins, $(0.5 \mathrm{~nm})^{3}$ voxels, $2 \times 2 \times 2$ binning, then up-sampled to original resolution. Score images (left column) are sum of 
middlemost 9 XZ-slices. (a) $1^{\text {st }} \mathrm{PC}$, which is the mean, (b) $2^{\text {nd }} \mathrm{PC}$, describing Cr-rich precipitates, and (c) $3^{\text {rd }} \mathrm{PC}$, describing $\mathrm{Fe}(\mathrm{Ti}, \mathrm{Al})$ precipitates. Arrows indicate a small precipitate at the edge of the volume.
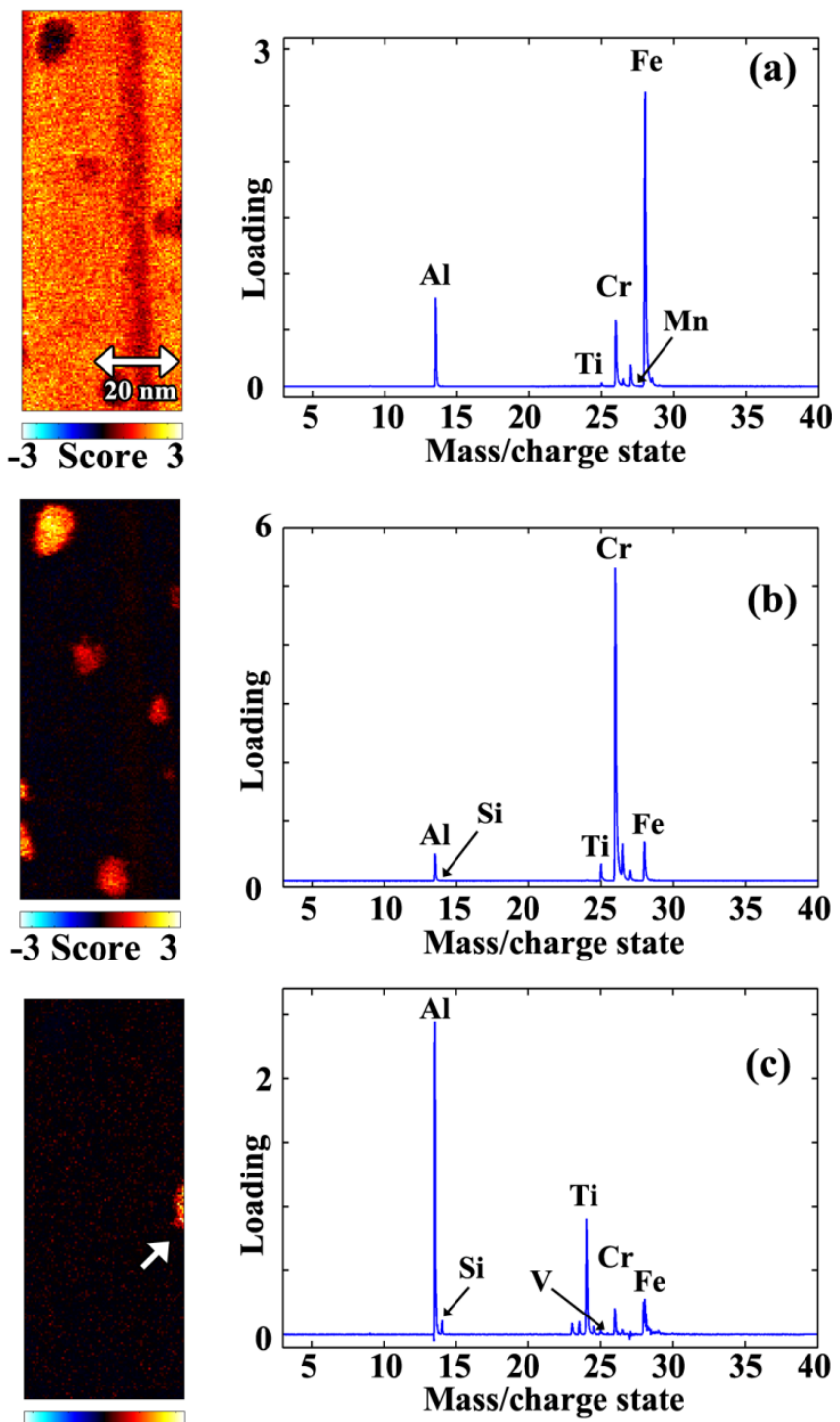

-2 Score 2

Figure 3: SS-PCA of sample aged at $475^{\circ} \mathrm{C}$ for $3600 \mathrm{~h}, 3$ to $40 \mathrm{u}, 0.02 \mathrm{u}$ bins, $(0.5 \mathrm{~nm})^{3}$ voxels, $2 \times 2 \times 2$ binning for PCA and then up-sampled to original resolution. Score images (left column) are sum of middle-most 9 XZ-slices. (a) $1^{\text {st }}$ SS component, which is the matrix. (b) $2^{\text {nd }}$ SS 
component, describing Cr-rich $\alpha^{\prime}$ precipitates. (c) $3^{\text {rd }}$ SS component, describing Fe(Ti,Al) $\beta^{\prime}$ precipitates. Arrows indicate a small precipitate at the edge of the volume.
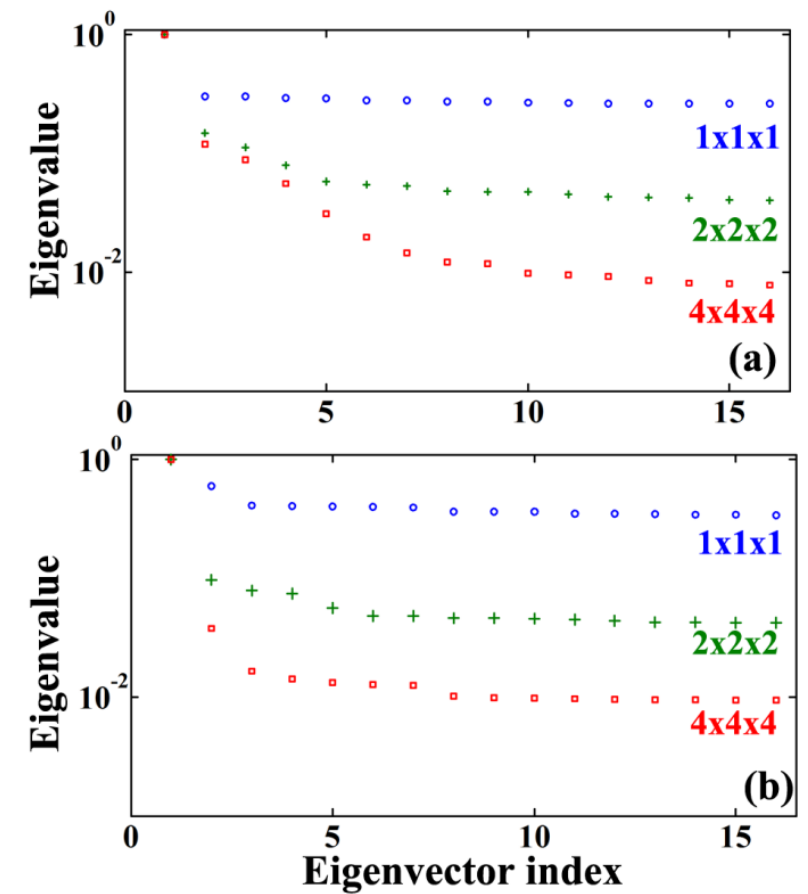

Figure 4: Normalized eigenvalue-eigenvector index plots for the samples aged at (a) $435^{\circ} \mathrm{C}$ for $3600 \mathrm{~h}$ and (b) $400^{\circ} \mathrm{C}$ for $3600 \mathrm{~h}$. Both processed using $(0.5 \mathrm{~nm})^{3}$ voxels, 3 to $40 \mathrm{u}, 0.02 \mathrm{u}$ bins. Voxel binning as indicated on each graph. 


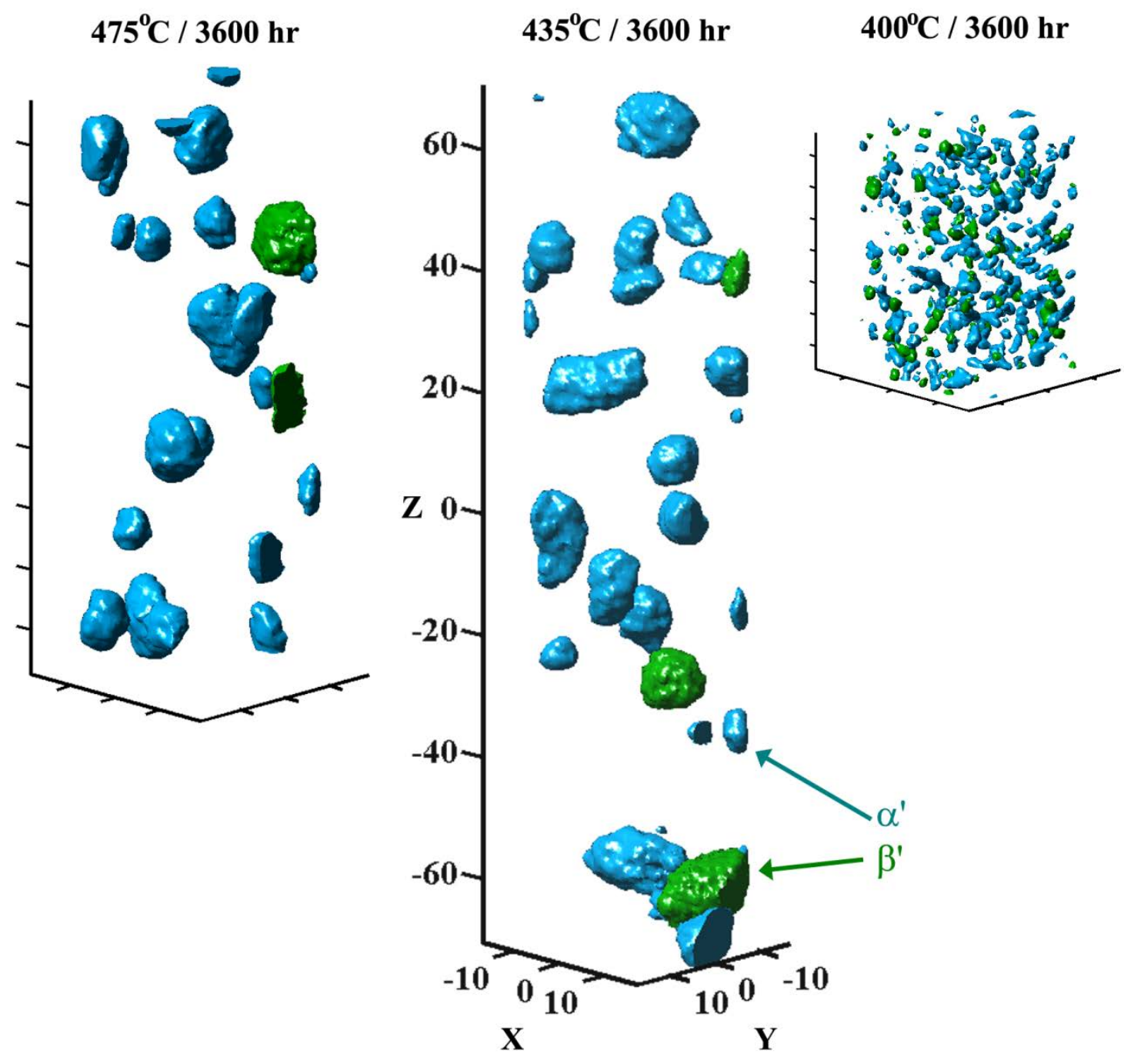

Figure 5: Thresholded and rendered isoscore surfaces for the SS-PCA components identified in the APT datasets from the three heat-treated (3600 h) alloys, (a) $475^{\circ} \mathrm{C}$, (b) $435^{\circ} \mathrm{C}$, and (c) $400^{\circ} \mathrm{C}$. All renderings are to approximately the same scale, indicated in nanometers. Precipitate populations are designated as follows: Cyan, $\mathrm{Cr}$-rich $\alpha^{\prime}$; green, $\mathrm{Fe}(\mathrm{Ti}, \mathrm{Al})-$ rich $\beta^{\prime}$ precipitates. All datasets processed using $(0.5 \mathrm{~nm})^{3}$ voxels, 3 to $40 \mathrm{u}, 0.02 \mathrm{u}$ bins, $2 \times 2 \times 2$ with up-sampling back to original resolution.

\section{Tables}


Table I: Sample conditions, APT reconstruction parameters, and MVSA parameters. Gb calculations assume 1851 channels, 8 bytes / element.

\begin{tabular}{cccccc}
$\begin{array}{c}\text { Temperature } \\
\left({ }^{\circ} \mathrm{C}\right)\end{array}$ & $\begin{array}{c}\text { Time } \\
(\mathrm{hr})\end{array}$ & $\begin{array}{c}\text { Number of } \\
\text { ions }\end{array}$ & $\begin{array}{c}\mathrm{X} \times \mathrm{Y} \times \mathrm{Z} \text { extent } \\
(\mathrm{nm})\end{array}$ & $\begin{array}{c}\text { Gb at } \\
(0.5 \mathrm{~nm})^{3}\end{array}$ & $\begin{array}{c}\text { Gb using } \\
\text { sparse data }\end{array}$ \\
\hline 400 & 3600 & $1.5 \times 10^{6}$ & $33 \times 33 \times 38$ & 5 & 0.02 \\
435 & 3600 & $6.3 \times 10^{6}$ & $35 \times 35 \times 141$ & 20 & 0.09 \\
475 & 3600 & $5.2 \times 10^{6}$ & $38 \times 38 \times 95$ & 16 & 0.07
\end{tabular}

